Spin Physics (SPIN2014)

International Journal of Modern Physics: Conference Series

Vol. 40 (2016) 1660079 (6 pages)

(C) The Author(s)

DOI: $10.1142 / \mathrm{S} 201019451660079 \mathrm{X}$

\title{
Test of Time-Reversal Invariance at COSY (TRIC)
}

\author{
D. Eversheim \\ Helmholtz Institut für Strahlen- und Kernphysik, \\ University Bonn, Bonn, D-53115, Germany \\ evershei@hiskp.uni-bonn.de \\ Yu. Valdau \\ Institut für Kernphysik, \\ Forschungszentrum Jülich, Jülich, D-52425, Germany \\ y.valdau@fz-juelich.de \\ B. Lorentz \\ Institut für Kernphysik, \\ Forschungszentrum Jülich, Jülich, D-52425, Germany \\ b.lorentz@fz-juelich.de
}

Published 29 February 2016

\begin{abstract}
At the Cooler Synchrotron COSY a novel (P-even, T-odd) null test of time-reversal invariance to an accuracy of $10^{-6}$ is planned as an internal target transmission experiment. The parity conserving time-reversal violating observable is the total cross-section asymmetry $\mathrm{A}_{\mathrm{y}, \mathrm{xz}}$. This quantity is measured using a polarized proton beam with an energy of $135 \mathrm{MeV}$ and an internal tensor polarized deuteron target from the PAX atomic beam source. The reaction rate will be determined by the lifetime of the beam. Consequently, the accuracy of the current measurement of the circulating proton beam is crucial for this experiment. Thus, the cooler synchroton ring serves as an ideal forward spectrometer, as a detector, and an accelerator.
\end{abstract}

Keywords: Time reversal invariance; internal total cross-section measurement.

PACS numbers: 11.30.Er, 13.85.Lg, 13.88.te, 29.20.Dh, 29.27.Fh

\section{Introduction}

The Standard Model ${ }^{1}$ of fundamental particles is based on a concept of symmetry. Therefore, searches for processes with a symmetry violation are an important tool to track down physics beyond the Standard Model (SM). This physics seems necessary in order to explain the Baryon Asymmetry i.e. matter-antimatter asymmetry of the

This is an Open Access article published by World Scientific Publishing Company. It is distributed under the terms of the Creative Commons Attribution 3.0 (CC-BY) License. Further distribution of this work is permitted, provided the original work is properly cited. 
Universe $^{2}$ (BAU). A ratio of the baryon-antibaryon difference over the number of cosmic background radiation photons, the baryon asymmetry parameter, can be derived to be $6 \cdot 10^{-10}$ from the WMAP and COBE missions. ${ }^{3}$ Up to now only CP violating processes are associated with matter antimatter asymmetry. These effects are incorporated into the SM using a theta term in the Lagrangian of quantum chromodynamics or a phase in the Cabibbo-Kobayashi-Maskawa matrix for three generations of quarks. The baryon asymmetry parameter is expected within the SM to be of the order of $10^{-18}$. Therefore, physics beyond the SM seems to be necessary to explain the BAU. Presently no processes are discovered which violate CPT symmetry. Hence, assuming CPT symmetry, CP violation implies a $\mathrm{T}$ violation. The $\mathrm{T}$ symmetry violation has been discovered in $\mathrm{K}$ and $\mathrm{B}$ meson systems, but has never been observed in a system of hadrons.

An EDM of an elementary particle simultaneously violates both $\mathrm{P}$ and $\mathrm{T}$ invariance and serves as a very sensitive probe for the physics beyond the SM. Assuming CPT symmetry it is possible to link an EDM of elementary particle to a $\mathrm{T}$ invariance and hence $\mathrm{CP}$ violation. At present the most sensitive test in this respect is the upper limit on an electric dipole moment of the neutron ${ }^{4}\left(1.6 \cdot 10^{-26} \mathrm{ecm}\right)$, which implies a strength of PT-violation of $\mathrm{g}_{\mathrm{PT}} \sim 10 .^{-11}$ This value seems already too small to explain the BAU. Thus, a different channel, for which $\mathrm{P}$ holds and only $\mathrm{T}$ is violated seems to be worth while to be explored. In this case an upper limit for $\mathrm{g}_{\mathrm{T}}<10^{-4}$ can be derived ${ }^{5}$ from the n-EDM.

The TRIC experiment ${ }^{6}$ will test a P-even T-odd interaction in a double polarized proton-deuteron scattering. Figure 1 shows the situation in the c.m.-system, with: (a) The basic system is shown. (b) The time reversal operation is applied (momenta and spins are reversed and the particles are exchanged). In order to have a direct comparison between situation (a) and (b), two rotations $R_{y}$ or $R_{x}$ by $180^{\circ}$ about

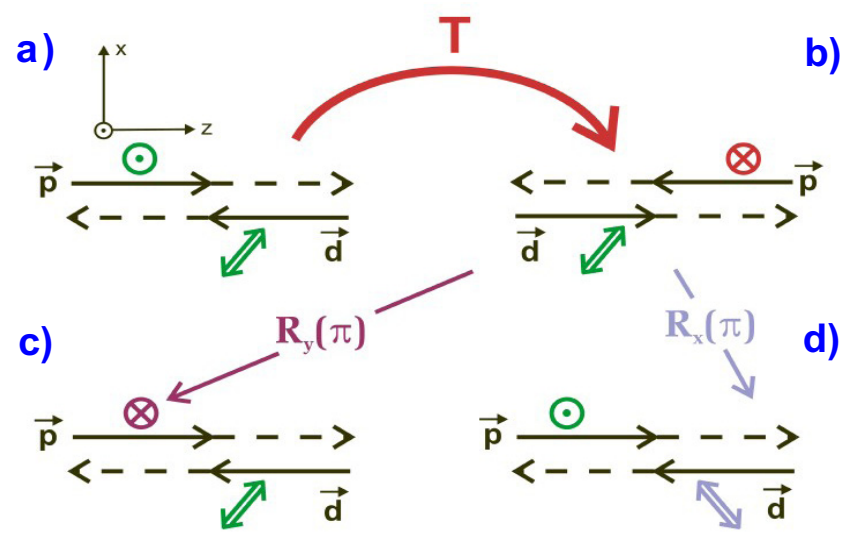

Fig. 1. Scheme of a time-reversed situation that is prepared by either a proton or a deuteron spin-flip. $\odot$ Proton spin up (y-direction), $\otimes$ Proton spin down, $\Leftrightarrow$ Deuteron tensor polarization. 
the $\mathrm{y}$ - or $\mathrm{x}$-axes are applied, leading to the situations c) and d), respectively. This is allowed, since the scattering process is invariant under spatial rotations.

It was shown ${ }^{7}$ that the total cross section of scattering a vector polarized proton beam off a tensor polarized deuterium target is a true P-even T-odd null observable, i.e. the total correlation $A_{y, x z}$. To conduct a null experiment has the advantage that systematic errors can be identified by switching off an essential beam and/or target parameter i.e. the polarization. Then, the result of the measurement has to be zero. In order to keep in general errors as small as possible, the experiment should be performed at an energy for which its sensitivity is highest. Beyer ${ }^{8}$ estimated this energy to be about $135 \mathrm{MeV}$ from the mass of the $\rho$-meson involved in the P-even T-odd process.

In the TRIC experiment a vector polarized proton beam will interact with an internal tensor polarized deuterium target placed in one of the straight sections of the COSY accelerator. In this experiment COSY will serve not only as an accelerator but also as an ideal zero degree spectrometer allowing for the application of the optical theorem, which relates the forward scattering amplitude to the total cross section. ${ }^{9}$ These total cross section measurements are inherently free from uncertainties due to final state interactions arising in many other experiments with nuclear beams that test fundamental symmetries. ${ }^{10}$ The total cross section of the double polarized proton-deuteron interaction will — depending on the spin setting of protons and deuterons - result in slopes of different steepness of the circulating COSY beam current. Using this novel method to measure total cross-sections and the simplest spin-1/2 - spin-1 system it is possible to improve an upper limit on the strength of the P-even T-odd interaction by an order of magnitude compared to the value previously discussed in literature. ${ }^{11}$

\section{The Experimental Set-Up}

The set-up is depicted in Fig. 2.

In contrast to external total cross-section measurements the TRIC experiment constitutes an internal measurement. Precise external total cross-section measurements are conducted either as transmission experiments or, if the optical theorem is utilized, by measuring the forward scattering amplitude. In the first case the accuracy is limited by the mutual calibration of the involved detectors with respect to all possible particles, energies and solid angles. In the latter case the accuracy is limited by the statistics, as the ideally allowed solid angle in forward direction should be zero. In addition, the calibration problem persists.

Performing the experiment internally, has the advantage that only the current measurement has to be utmost accurate. In principle this is a transmission experiment too, since the losses due to scattering in the target-cell are measured by the decrease of the beam intensity as the beam circulates through the target about a million times per second. 


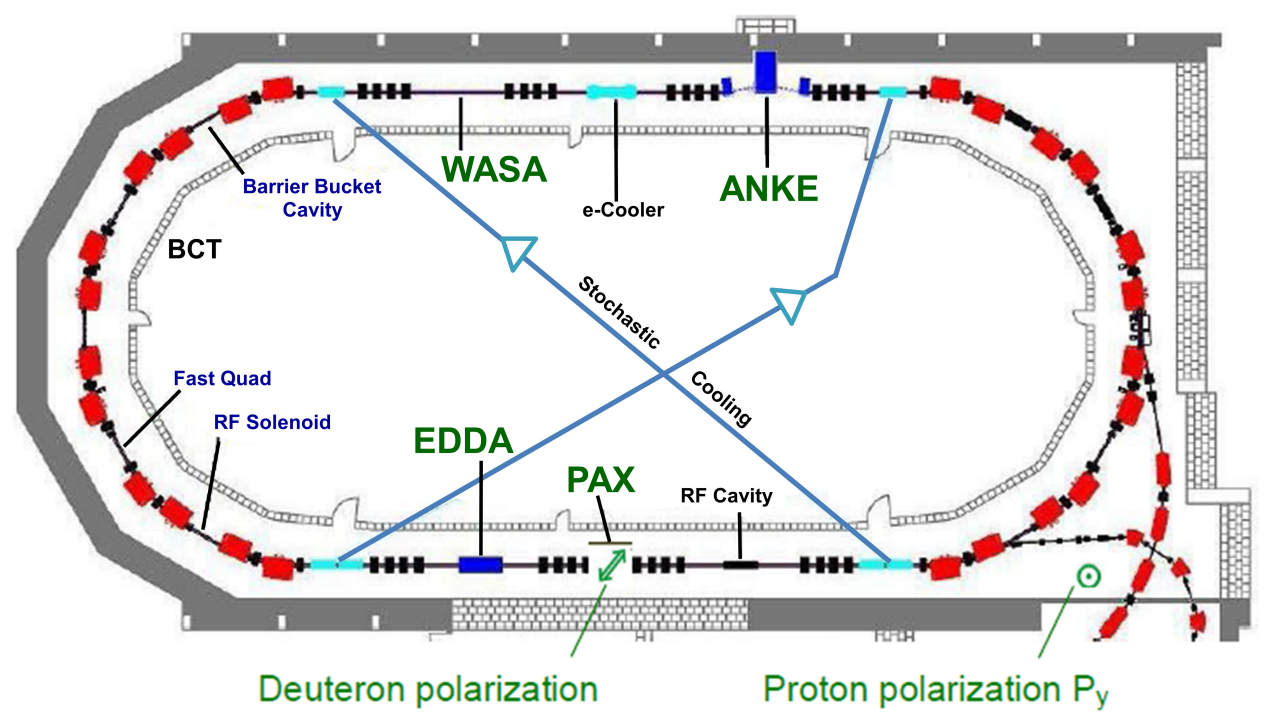

Fig. 2. The TRIC experiment will take place at the PAX target location.

Recently the COSY staff succeeded in providing polarized bunched beams with sufficiently long lifetimes. This allows for simpler and therefore more accurate measurement devices (integrated current transformer) compared to the presently installed device for measuring coasting (continuous DC) beams in COSY. In turn, an increased measurement accuracy permits shorter spin-flip periods and consequently reduces systematic errors due to drifts. In this respect we expect a reduction of systematic error contributions by more than an order of magnitude. The currently available Beam Current Transformer ${ }^{12}$ (BCT) together with its readout scheme at COSY limits the possible accuracy of beam current measurements for the TRIC experiment. Furthermore, the performance of this instrument for the bunched beam was never studied nor calibrated.

\section{Error Considerations}

In Table 1 all observables in $\vec{p}-\vec{d}$ scattering are listed.

All observables of Table 1 in lines 1 and 5 cancel if $A_{y, x z}$ is calculated from a proton polarization that is flipped each time the ring is filled. All observables with respect to the proton polarization $4 \mathrm{P}_{\mathrm{x}}$ and $\mathrm{P}_{\mathrm{z}}$ cancel too, since only the proton polarization $\mathrm{P}_{\mathrm{y}}$ is an eigenvector in the ring (the average of $\mathrm{P}_{\mathrm{x}}$ and $\mathrm{P}_{\mathrm{z}}$ should be $<$ $10^{-8}$ in a 30 days run). This is also true in Table 1 for lines $2,4,6$, and 8 .

In the remaining lines 3 and 7 all quantities with a hat cancel, because they are not $R_{z}$-even. $A_{y, x}$ and $A_{y, y z}$ violate parity conservation. Therefore, since $\vec{p}-\vec{d}$ scattering is an elementary process, these quantities are expected to be of the order of $10^{-7}$, even if parity is violated. Thus, besides our quantity of interest $\mathrm{A}_{\mathrm{y}, \mathrm{xz}}$, only 
Table 1. Polarization observables of the total cross-section in $\vec{p}-\vec{d}$ scattering. The first index refers to the proton polarization, the second and third index refers to the deuteron vector- and tensor-polarization. All quantities with a hat cancel, since they are $\mathrm{R}_{\mathrm{z}}$-odd. All quantities which are doubly underlined are $\mathrm{P}_{\mathrm{z}}$-odd.

\begin{tabular}{|c|c|c|c|c|c|c|c|}
\hline Li & \multicolumn{5}{|c|}{ Observable } & & \multirow{2}{*}{$\begin{array}{l}\text { Line cancels because of: } \\
\text { protonspinflip }\end{array}$} \\
\hline 1 & $\mathrm{I}_{\mathrm{o}, \mathrm{o}}$ & $\underline{\underline{\underline{\hat{A}}}} \underline{\underline{\underline{x} . x}}$ & $A_{o, y}$ & $\underline{\underline{\underline{\hat{A}_{0, z}}}}$ & & & \\
\hline 2 & $\underline{\underline{\hat{A}}} \underline{\underline{\hat{x}_{0}}}$ & $\mathrm{~A}_{\mathrm{x}, \mathrm{x}}$ & $\underline{\underline{A_{x, y}}}$ & $\underline{\underline{\hat{A}}}_{x, z}$ & & & $P_{x}$ negligible for protons \\
\hline 3 & $\underline{\underline{\hat{\hat{\hat{A}}}}}_{\mathrm{y}, \mathrm{o}}$ & $\underline{\underline{\mathrm{A}_{\mathrm{y}, \underline{x}}}}$ & $\mathrm{~A}_{\mathrm{y}, \mathrm{y}}$ & $\underline{\underline{\hat{\hat{A}}_{\mathrm{y}, z}}}$ & & & \\
\hline 4 & $\underline{\underline{\mathrm{A}_{z, 0}}}$ & $\underline{\underline{\hat{A}}}_{z, \mathrm{x}}$ & $\underline{\underline{\underline{\hat{A}}}}_{\underline{\underline{z y}}}$ & $\mathrm{~A}_{\mathrm{z}, \mathrm{z}}$ & & & $P_{z}$ negligible for protons \\
\hline 5 & $A_{0, x x}$ & $\mathrm{~A}_{\mathrm{o}, \mathrm{yy}}$ & $\mathrm{A}_{\mathrm{o}, \mathrm{zz}}$ & $\underline{\underline{\mathrm{A}_{0 . x y}}}$ & $\underline{\underline{\hat{A}}}_{\underline{0, y z}}$ & $\underline{\underline{\hat{A}}}_{0, x z}$ & protonspinflip \\
\hline 6 & $\underline{\underline{\hat{\hat{A}}}} \underline{\underline{x x}}$ & $\underline{\underline{\hat{A}_{x . y y}}}$ & $\underline{\underline{\hat{A}_{x, z Z}}}$ & $\underline{\underline{\hat{A}}}_{x, x y}$ & $\mathrm{~A}_{\mathrm{x}, \mathrm{yz}}$ & $\underline{\underline{\mathrm{A}_{x, x z}}}$ & $P_{x}$ negligible for protons \\
\hline 7 & $\underline{\underline{\hat{\hat{A}}}}_{y, x x}$ & $\underline{\underline{\hat{\hat{A}}}}_{\mathrm{y}, \mathrm{yy}}$ & $\underline{\underline{\hat{\hat{A}}}}_{y, z z}$ & $\underline{\underline{\hat{\hat{A}}}}_{\underline{y, x y}}$ & $\underline{\underline{\mathrm{A}_{\mathrm{y}, y z}}}$ & $\mathrm{~A}_{\mathrm{y}, \mathrm{xz}}$ & \\
\hline 8 & $\underline{\underline{\mathrm{A}_{z, x \mathrm{x}}}}$ & $\underline{\underline{\mathrm{A}_{z . y y}}}$ & $\underline{\underline{\mathrm{A}_{z, z z}}}$ & $\mathrm{~A}_{z, \mathrm{xy}}$ & $\underline{\underline{\hat{\hat{A}}}}_{z, \mathrm{yz}}$ & $\underline{\underline{\hat{A}_{z, x z}}}$ & $P_{z}$ negligible for protons \\
\hline
\end{tabular}

$\mathrm{A}_{\mathrm{y}, \mathrm{y}}$ "survives". Since $\mathrm{A}_{\mathrm{y}, \mathrm{y}}$ in $\overrightarrow{\mathrm{p}}-\overrightarrow{\mathrm{d}}$ scattering is not known at $135 \mathrm{MeV}$ it has to be determined in a dedicated measurement.

The effect of $A_{y, y}$ in $\vec{p}-\vec{d}$ scattering is expected to be small, because (i) there must be a deuteron vector polarization in the target, and (ii) there must be a misalignment between the COSY beam direction and the deuteron beam, so that a deuteron vector polarization in the atomic beam source is able to generate a $\mathrm{P}_{\mathrm{y}}$ deuteron vector polarization in the target-cell. The deuteron vector polarization can be adjusted to be close to zero in the atomic beam source. The deuteron vector polarization can be continuously monitored in the dump of the atomic beam source.

\section{Error Path}

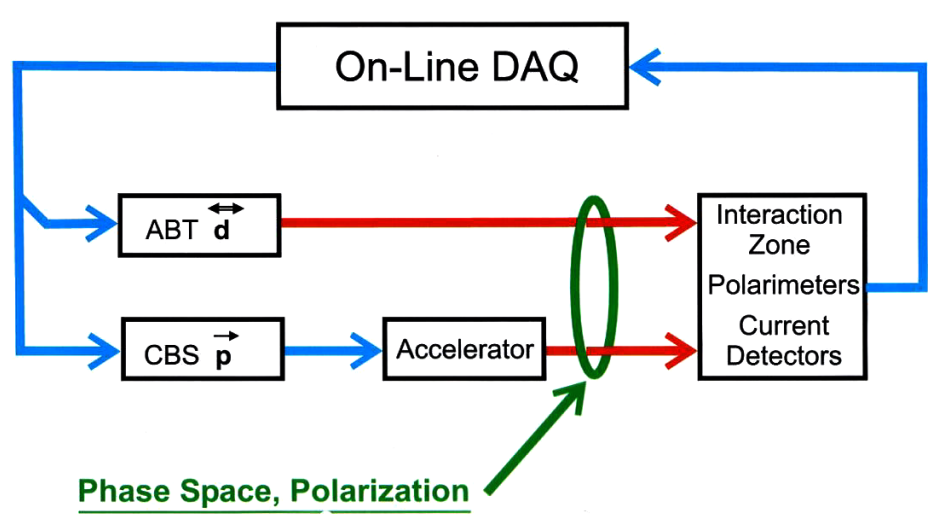

Fig. 3. Errors are introduced to the experiment by spin-flip correlated changes of beam and/or target. 
Assuming the deuteron vector polarization can be limited to 0.01 , and the deuteron source and the proton beam can be aligned to better than $0.1^{\circ}$, then a false deuteron vector polarization of $\mathrm{P}_{\mathrm{y}}<2 \cdot 10^{-5}$ results. If $\mathrm{A}_{\mathrm{y}, \mathrm{y}}$ in $\overrightarrow{\mathrm{p}}-\overrightarrow{\mathrm{d}}$ scattering is $<0.05$ the error contribution is $<10^{-6}$.

The question how errors are introduced to the experiment in general is answered by Fig. 3 .

As the TRIC experiment can be considered as a correlation experiment in the time-domain, the interaction rate and thereof the slope of the decreasing beam current is changed only, if the beam properties change correlated with the spin-flip of beam and/or target. As a consequence, the phase space including the polarizations of beam and target have to be under control. In this respect polarimeters, beam position monitors and redundancy become important. For instance: The beam/target spin configuration ++ and -- as well as +- and -+ should give the same result. These inherent cross-checks together with the unique P-even T-odd true null observable $\mathrm{A}_{\mathrm{y}, \mathrm{xz}}$ can allow to measure this quantity within 30 days to an accuracy of $10^{-6}$.

\section{Acknowledgment}

The enduring and conscientious work of the COSY team while developing the bunched beam for TRIC is greatly acknowledged.

\section{References}

1. J. M. Cline, arXiv: hep-ph/0609145.

2. A. Sakharov, Pisma Zh. Eksp. Teor. Fiz. 5, 32 (1967).

3. D. N. Spergel et al., arXiv: astro-ph/0302209v3.

4. C. A. Baker et al., Phys. Rev. Lett. 97, 131801 (2006).

5. W. C. Haxton, A. Höring and M. J. Musolf, Phys. Rev. D 50, 3422 (1994).

6. P. D. Eversheim, B. Lorentz, Yu. Valdau for the PAX collaboration, COSY Proposal \#215.

7. H. E. Conzett, Phys. Rev. C 48, 423 (1993).

8. M. Beyer, Nucl. Phys. A 560, 895 (1993).

9. C. Bourrely, E. Leader and J. Soffer, Phys. Rep. 59, 95 (1980).

10. J. Murata et al., Hyperfine Interact. 225, 193 (2014).

11. P. R. Huffmann et al., Phys. Rev. C 55, 2684 (1997).

12. Bergoz Instrumentation, Parametric Current Transformer, Instruction Manual (1987). 